\title{
Superficial Ulcerating Rheumatoid Necrobiosis Associated with Methotrexate Use in a Patient with Rheumatoid Arthritis
}

\author{
Authors: \\ Austin Cusick,, Amandeep Goyal, ${ }^{2}$ Ashley H. Merten, ${ }^{3}$ Andrew Virata, ${ }^{3}$ \\ Rahul Sehgal, 3 *Pankaj Bansal ${ }^{3}$ \\ 1. Ohio University Heritage College of Osteopathic Medicine, Athens, Ohio, USA \\ 2. Marietta Memorial Hospital, Marietta, Ohio, USA \\ 3. Mayo Clinic Health System, Eau Claire, Wisconsin, USA \\ *Correspondence to bansal.pankaj@mayo.edu
}

Disclosure: $\quad$ The authors have declared no conflicts of interest.

Acknowledgements: The authors would like to thank the patient for allowing her case to be presented for educational purposes.

Received:

13.03 .20

Accepted:

09.06.20

Keywords:

Methotrexate, rheumatoid arthritis (RA), superficial ulcerating rheumatoid necrobiosis (SURN).

Citation:

EMJ. 2020;5[3]:39-44.

\section{Abstract}

Methotrexate, a disease-modifying antirheumatic drug, is fundamental to limiting progression in several rheumatic diseases such as rheumatoid arthritis (RA). However, methotrexate is also associated with various significant adverse effects. Of note, there are several dermatologic manifestations attributed to methotrexate therapy. In particular, accelerated nodulosis and panniculitis are linked to methotrexate therapy in the current literature. The authors present the case of a 55-year-old Caucasian female with seropositive erosive RA who developed superficial ulcerating rheumatoid necrobiosis (SURN), secondary to methotrexate therapy. The patient's treatment consisted of methotrexate discontinuation, topical clobetasol, and initiation of leflunomide as a replacement of methotrexate. Follow-up evaluation confirmed resolution of SURN over time and maintained low disease RA activity with leflunomide.

Few cases describe SURN in the setting of RA and there are currently no cases published that suggest methotrexate's possible role in SURN. Methotrexate-induced SURN is plausible in this case because of the correlation with therapy initiation and remission after therapy discontinuation. SURN has significant histological overlap with other methotrexate-induced dermatologic manifestations, allowing for a possible correlation. Most dermatological side effects of methotrexate are linked to a genetic predisposition of the HLA-DRB1 gene. Additionally, methotrexate's mechanism of action for rheumatologic disease paradoxically stimulates adenosine-1 receptors and activates neutrophil chemotaxis and phagocytosis. Adenosine-1 receptor stimulation is hypothesised to be the source of rheumatoid-accelerated nodulosis and possibly SURN. Furthermore, the location of manifestation, genetic predisposition, and comorbid features in the patient all possibly have a role in this unique dermatological side effect. 


\section{INTRODUCTION}

Rheumatoid arthritis (RA) is a systemic autoimmune condition prevalent in North America and Northern European populations with an incidence of $1 \%$. $^{1}$ It affects females disproportionately to males; the lifetime risk of development is $3.6 \%$ and $1.7 \%$, respectively. ${ }^{2}$ Several therapeutic regimens are available for RA under the categorisation of diseasemodifying antirheumatic drugs (DMARD). Early intervention with immediate initiation of DMARD therapy is recommended to prevent radiographic progression of the disease. ${ }^{3}$

Methotrexate (MTX) is one of the first-line DMARD used to treat RA. Several mechanisms of action have been suggested regarding MTX efficacy as a DMARD therapy. ${ }^{4}$ These mechanisms include inhibition of nucleotide synthesis causing precursor accumulation to a degree, but the specifics of each mechanism are unique. A proposed mechanism by Cronstein et al. ${ }^{4}$ includes the inhibition of 5aminoimidazole-4-carboxamide-ribonucleotide (AICAR) transformylase. This subsequently increases the concentration of AICAR, and more specifically, adenosine; adenosine then perpetuates an anti-inflammatory response., ${ }^{4,5}$ The other, more commonly accepted mechanism is the inhibition of dihydrofolate reductase and thymidylate synthase, which induces cellular depletion and inhibition of de novo purine synthesis. $^{5}$

MTX therapy has several well-documented adverse effects, with up to $20 \%$ of patients discontinuing usage in response to this. ${ }^{5}$ Various reported toxicities of MTX are grouped into cardiotoxicity, haematologic toxicity, nephrotoxicity, pulmonary toxicity, hepatotoxicity, and carcinogenic toxicity. ${ }^{5}$ Additionally, more common side effects that decrease patient tolerance are gastrointestinal in origin and include nausea, vomiting, and diarrhoea. ${ }^{5}$ MTX therapy is also responsible for several dermatologic adverse effects; ${ }^{6}$ some patients with RA beginning MTX therapy have reported accelerated nodulosis, with some cases not showing resolution after medication discontinuation. ${ }^{6}$ This side effect has been associated with HLA-DRB1 and activation of local adenosine receptors in genetically susceptible individuals. ${ }^{6}$ Furthermore, additional evidence suggests a correlation between MTX-induced accelerated nodulosis and subsequent panniculitis in these susceptible individuals. ${ }^{7}$

Superficial ulcerating necrobiosis, a rare entity that may accompany RA, can develop to become superficial ulcerating rheumatoid necrobiosis (SURN). ${ }^{8}$ Necrobiosis is a generalised descriptor that refers to skin without connective tissue fibres upon histological examination Histologically, these lesions are characterised by bordering palisading histiocytes, multinucleated cells, and epithelioid cells. ${ }^{8}$ Necrobiosis is often associated with necrobiosis lipoidica diabeticorum (NLD) because these lesions demonstrate collagen degradation. Other dermatopathological manifestations may be described histologically as necrobiosis such as granuloma annulare and rheumatic nodules. ${ }^{8}$ Physical examination of skin lesions may reveal a shallow ulcer formation, typically found on the legs; although, erythematous papular eruptions without ulceration have been reported. ${ }^{9}$ MTXinduced SURN has not been described in the current literature and warrants further discussion.

The authors herein present the case of a 55-year-old Caucasian female with seropositive erosive RA who developed SURN after initiation of MTX therapy.

\section{CASE DESCRIPTION}

A 55-year-old female with 20-year history of seropositive (rheumatoid factor and anti-CCP positive) erosive RA on MTX therapy presented with firm, yellow/violaceous, depressed papules and plaques with central telangiectasias on the bilateral shins, calves, and dorsal feet. Some lesions had raised edges, and sizes ranged from $5 \mathrm{~mm}$ to $3 \mathrm{~cm}$ (Figure 1). Her eruption had started upon initiation of MTX therapy longer than 10 years ago, and she reported increased size and number of plaques in subsequent years. She had chosen not to pursue evaluation for the rash priorly as it had been asymptomatic, but it was now becoming increasingly disfiguring. She had had low RA disease activity for the previous few years with a Clinical Disease Activity Index (CDAl score ranging from 2-5 and Disease Activity Score-28 and C-reactive protein (DAS-28-CRP) scores ranging from 1.63-1.93 over the past year on MTX $25 \mathrm{mg}$ 


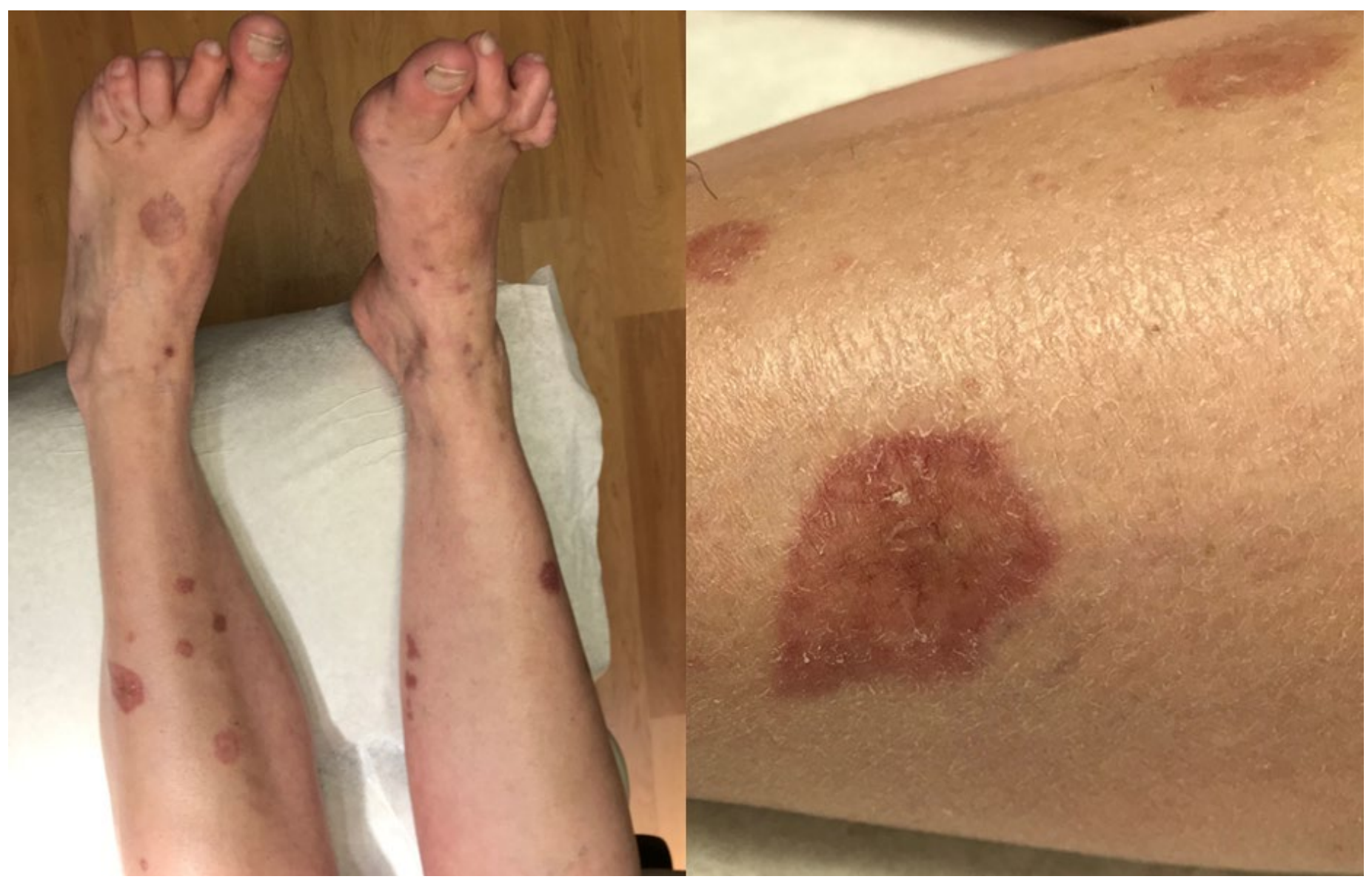

Figure 1: Initial presentation with firm, yellow/violaceous, depressed papules and plaques with central telangiectasias on the bilateral shins, calves, and dorsal feet. Some lesions presented with raised edges and sizes ranging from $5 \mathrm{~mm}$ to $3 \mathrm{~cm}$.

subcutaneous weekly monotherapy. She did not have any subcutaneous rheumatoid nodules.

A skin biopsy demonstrated dermal palisading granulomas with central necrobiotic collagen (Figure 2). There were several associated lymphocytes, plasma cells, neutrophils, and eosinophils. Periodic acid-Schiff and acid-fast bacilli stains were negative for infectious organisms. A colloidal iron stain did not demonstrate increased dermal mucin (Figure 2). No vasculitis was identified on the histopathology. These findings were thought to be compatible with palisading granulomatous dermatitis with necrobiotic collagen, consistent with SURN.

Given the historical suggestion by the patient about the onset of the eruption coinciding with initiation of MTX, her MTX was discontinued and she was started on leflunomide to treat her RA. She was also started on topical clobetasol
0.05\% ointment. After 2 months, the eruption significantly improved, with all old lesions fading and no presentation of new lesions. Topical corticosteroids were discontinued and on 6-month follow-up the eruption had resolved, with some residual post-inflammatory hyperpigmentation despite topical corticosteroid cream discontinuation several months previously (Figure 3). The patient maintained low RA disease activity on leflunomide.

SURN has been reported in patients with RA, but the authors found no reported cases of MTX-associated SURN in the literature. Despite this, MTX-induced accelerated nodulosis is a well-reported phenomenon. Given the strong timeline association of rash onset after initiation of MTX therapy and the improvement in rash after discontinuing MTX, it is highly probable that the SURN was caused by methotrexate in this patient. 

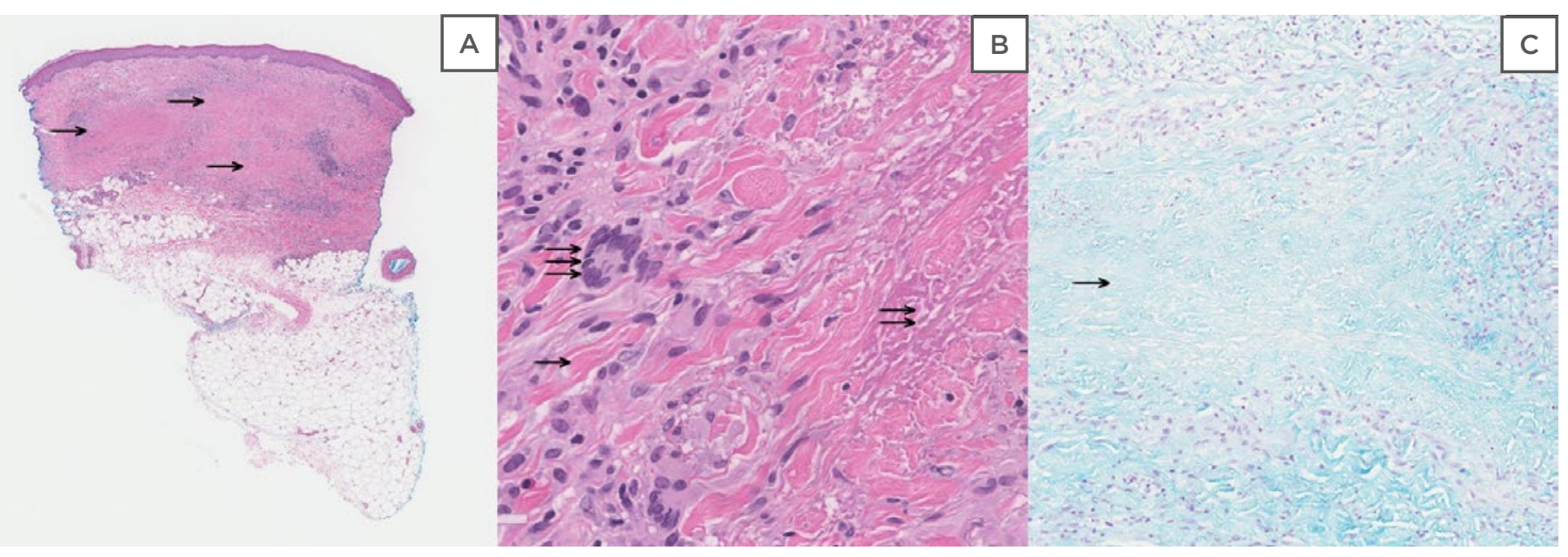

Figure 2: A) $4 \mathrm{~mm}$ punch biopsy of the skin; haematoxylin and eosin stain (low power, 2x magnification) demonstrates palisaded granulomatous dermal inflammation (single arrows). B) Haematoxylin and eosin stain (high power, 40x magnification) demonstrates intact peripheral collagen bundles (single arrow), necrobiotic collagen (double arrow), and surrounding palisaded granulomatous dermal inflammation with multinucleated histiocytes (triple arrow). C) Colloidal iron stain (intermediate power, 10x magnification) is negative for increased dermal mucin in zones of necrobiotic collagen (single arrow).

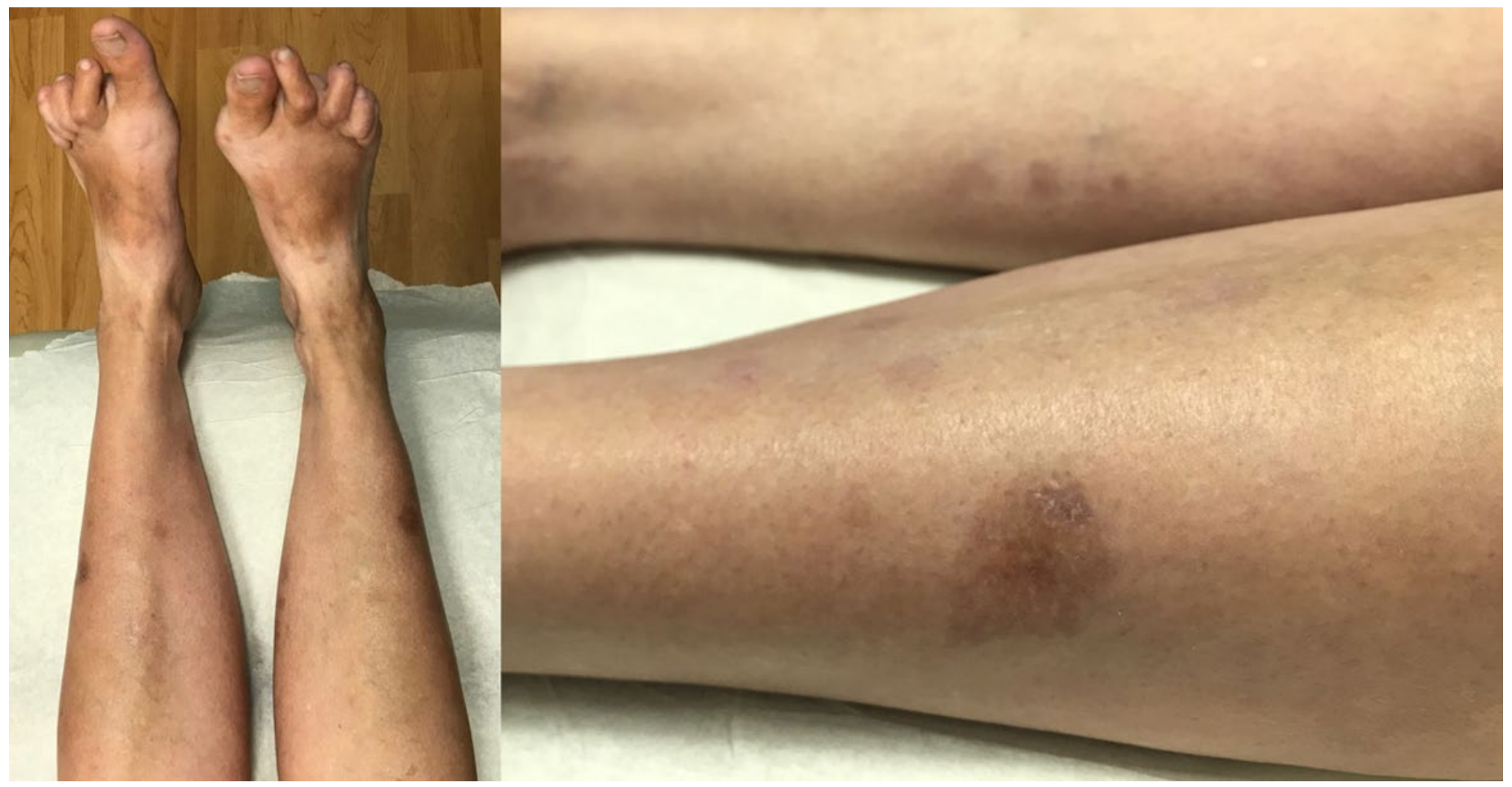

Figure 3: Resolution of eruption is shown, with residual post-hyperpigmentation changes 6 months after discontinuing methotrexate. 


\section{DISCUSSION}

MTX is a widely used DMARD and is often the first-line treatment for RA. MTX has been well known to cause accelerated nodulosis in patients with RA and there are some reports showing the prevalence of nodulosis is as high as $8 \%$ amongst patients treated solely on MTX.10 Additionally, accelerated nodulosis induced by MTX has been reported in patients with duration of therapy ranging from 3 months to 12 years after treatment initiation." Therefore, the role of MTX is plausible in this described case.

Several extra-articular cutaneous manifestations of RA have levels of overlap based on histology. Generalised necrobiosis is evident through focal degeneration of connective tissue and necrobiosis can be found in several other pathologies including rheumatoid nodules, granuloma annulare, and NLD. ${ }^{2}$ Histology analysis shows disrupted collagen fibres with collagen hyalinisation. The mechanism of necrobiosis is linked to immune complex deposition, which leads to vasculitis and subsequent tissue remodelling by matrix metalloproteinases (MMP). ${ }^{12}$

Rheumatoid nodules present in RA can show evidence of necrobiosis in their histological description. Classically, they are subcutaneous, firm, mobile nodules found predominately on, but not limited to, extensor surfaces of the body. ${ }^{13}$ Rheumatoid nodules are classified histologically into three distinct parts, as described by Yamamoto et al: $:^{13}$ the first descriptor is a core of necrosis with eosinophilic infiltrate and the second and third histologic findings include a palisading zone and chronic inflammatory cells infiltrating the perivascular space. ${ }^{13}$ Cells that primarily predominate in the palisading zones include activated macrophages and T-cell lymphocytes. These cells are thought to be responsible for the necrobiosis. ${ }^{14}$ Necrobiosis and the subsequent breakdown of Type 1 collagen is thought to be secondary to TNF- $\alpha$ cytokine-induced activation of MMP1 and MMP-3. ${ }^{15}$ Ulceration is rarely described in the setting of rheumatoid nodules but could be plausible in patients with comorbid vascular insufficiency. ${ }^{13}$

SURN is rarely described in RA. Jorizzo et al. ${ }^{8}$ describe two cases of patients who presented with leg lesions similar to the patient described in this report. Both cases had aggressive RA and developed focal ulcerating lesions in the lower legs; however, both patients' lesions were classified as NLD.16 This seemed unlikely because these patients had rheumatoid nodules, mild rheumatoid vasculitis, and high-titre rheumatoid factor levels. Therefore, the authors proposed a distinct entity of patients with a histologically similar process related to subclinical vasculitis and immune complex deposition. ${ }^{16}$

Using information gathered from similar, overlapping skin manifestations in RA, it is possible to deduce a pathway for further exploration of MTX-induced SURN. MTX-induced accelerated nodulosis also has some overlap with the above described patient's presentation in terms of both histology and history. Accelerated nodulosis has been documented across a variety of dosages and durations of treatment." Furthermore, it has a histological presentation of palisading granulomas and focal necrobiosis similar to the above described case. ${ }^{13}$ Since MTXinduced accelerated nodulosis and panniculitis are linked to patients expressing $H L A-D R B 1$, it is important to note there is a certain genetic susceptibility present in these patients. ${ }^{6,7}$ Therefore, the hypothesised mechanism of MTX's role in the pathogenesis of SURN could be similar.

MTX has been hypothesised to induce rheumatoid nodules through its mechanism of action. Understanding this mechanism helps isolate the potential cause behind the acceleration of rheumatoid nodules. As previously mentioned, the therapeutic mechanism of action for MTX as DMARD therapy is through the inhibition of AICAR transformylase. ${ }^{4}$ This causes an increase of AICAR, which inhibits conversion of adenosine monophosphate to inosine monophosphate. ${ }^{15}$ Additionally, AICAR increases adenosine to excessive levels. This stimulates low affinity adenosine-2 receptors located on inflammatory cells like neutrophils, monocytes, lymphocytes, and basophils, halting synthesis of cytokines and other inflammatory molecules. ${ }^{4}$ Side-effect manifestation is linked to increased extracellular adenosine-stimulating high-affinity adenosine-1 (A1) receptors. ${ }^{4,13}$ A1 receptors are present on macrophages and neutrophils and on these white blood cells they enhance immunologic functionality through 
heightened chemotaxis and phagocytosis of immunoglobulins. ${ }^{4,13}$ While histological examination for IgM was not investigated in this case, past reports have shown evidence of IgM deposition in dermal blood vessels with both SURN and rheumatoid nodules.,13 Activated macrophages and neutrophils through A1 receptors may then more readily phagocytose IgM complexes within dermal blood vessels. This is the likely mechanism behind DMARD therapy and is the possible manifestation of accelerated nodulosis or SURN. ${ }^{13}$

While correlative data and overlapping disease characteristics may help support the notion of SURN development from MTX therapy, direct causality could not be attributed between manifestation and pharmacologic agent. This case review is limited by the lack of a rechallenge with MTX. Re-introducing MTX would allow observation for the redevelopment of SURN. Reformation of SURN after a re-challenge would provide significant strength behind the hypothesis that the dermatologic manifestation was secondary to drug therapy. While a rechallenge analysis would be beneficial, subjecting a patient to possible redevelopment of the disease would be unethical in the clinical setting. In addition, re-challenging one patient in this study would not provide the statistical power necessary to consider the possible harms of evaluation. Future investigation and the details of this report will allow future examiners to assemble a larger sample size and appropriately develop a more substantial re-challenge assay.

\section{CONCLUSION}

In the few cases describing SURN, the cases presented as shallow ulcers on the lower legs, commonly the pretibial area. These cases had significant overlap with NLD and were often considered as such. ${ }^{8}$ It is hypothesised that these lesions are formed from a combination of factors including leg location, possible presence of vasculitis, and possible comorbid vascular insufficiency. ${ }^{13}$ These factors, combined with MTX-stimulating inflammation or necrobiosis in a genetically susceptible individual via A1 receptors could be the plausible pathophysiology responsible for the described case. Nevertheless, further cases and research would help elucidate the possible role of MTX in the formation of SURN.

\section{References}

1. Silman A, Pearson J. Epidemiology and genetics of rheumatoid arthritis. Arthritis Res. 2002;4(Suppl 3):S265-72.

2. Crowson $\mathrm{C}$ et al. The lifetime risk of adult-onset rheumatoid arthritis and other inflammatory autoimmune rheumatic diseases. Arthritis Rheum. 2011:63(3):633-9.

3. Molina $E$ et al. Association of socioeconomic status with treatment delays, disease activity, joint damage, and disability in rheumatoid arthritis. Arthritis Care Res (Hoboken). 2015;67(7):940-6.

4. Cronstein B et al. The antiinflammatory mechanism of methotrexate. Increased adenosine release at inflamed sites diminishes leukocyte accumulation in an in vivo model of inflammation. J Clin Invest. 1993;92(6):2675-82.

5. Wang $W$ et al. Side effects of methotrexate therapy for rheumatoid arthritis: a systematic review. Eur J Med Chem. 2018;158:502-16.

6. Enginar $\mathrm{E}$ et al. accelerated nodulosis in a patient with rheumatoid arthritis.
Arch Rheumatol. 2018;34(2):225-8.

7. Al Maashari R, Hamodat M. Methotrexate-induced panniculitis in a patient with rheumatoid arthritis. Acta Dermatovenerol Alp Pannonica Adriat. 2016;25(4):79-81.

8. Jorizzo J et al. Superficial ulcerating necrobiosis in rheumatoid arthritis. A variant of the necrobiosis lipoidicarheumatoid nodule spectrum? Arch Dermatol. 1982;118(4):255-9.

9. Chu P et al. The histopathologic spectrum of palisaded neutrophilic and granulomatous dermatitis in patients with collagen vascular disease. Arch Dermatol. 1994;130(10):1278-83.

10. Kerstens PJ et al. Accelerated nodulosis during low dose methotrexate therapy for rheumatoid arthritis. An analysis of ten cases. J Rheumatol 1992;19(6):867-71.

11. Patatanian E, Thompson D. A review of methotrexate-induced accelerated nodulosis. Pharmacotherapy 2002;22(9):1157-62.

12. Lynch J, Barrett T. Collagenolytic (necrobiotic) granulomas: part II-the 'red' granulomas. J Cutan Pathol. 2004;31(6):409-18

13. Yamamoto T. Cutaneous necrobiotic conditions associated with rheumatoid arthritis: important extra-articular involvement. Mod Rheumatol. 2013;23(4):617-22.

14. Wikaningrum $\mathrm{R}$ et al. Pathogenic mechanisms in the rheumatoid nodule: comparison of proinflammatory cytokine production and cell adhesion molecule expression in rheumatoid nodules and synovial membranes from the same patient. Arthritis Rheum. 1998;41(10):1783-97.

15. Ågren $M$ et al. Tumor necrosis factor- $\alpha$-accelerated degradation of Type I collagen in human skin is associated with elevated matrix metalloproteinase (MMP)-1 and MMP-3 ex vivo. Eur J Cell Biol. 2015;94(1):12-21.

16. Mangoni A et al. Methotrexate and cardiovascular protection: current evidence and future directions. Clin Med Insights: Therapeutics. 2017;9:1-12. 